\title{
Botanische Kostbarkeiten aus dem Iran
}

\author{
MiCHAEL JÄGER
}

\begin{abstract}
The flora of many regions in Iran is insufficiently known, and new species are still to be detected. During several field trips, Dionysia species, several geophytes and Araceae were found. Eminium jaegeri (Araceae), described in 2008, flowers in autumn. Another recently described species is Ophrys zagrica.
\end{abstract}

\section{Zusammenfassung}

Im Iran sind noch viele Gebiete floristisch unerforscht, so dass neue Arten entdeckt werden können. Auf mehreren Reisen in das Zagros-Gebirge wurden die Standortsbedingungen von Dionysia-Arten, diversen Geophyten und Araceen studiert. Dabei wurde ein neues herbstblühendes Aronstabgewächs gefunden, das 2008 als Eminium jaegeri veröffentlicht wurde. Ein weiterer Neufund war Ophrys zagrica.

\section{Reisen zu Naturstandorten kultivierter Pflanzen}

Schon seit über 20 Jahren werden im botanischen Garten Gießen Dionysia-Arten (Primulaceae) kultiviert. Es handelt sich dabei um Polsterpflanzen aus dem Iran und Afghanistan. Mit ihrem dichten Blütenflor erinnern sie an die in den Alpen heimischen Mannsschilder (Gattung Androsace), mit denen sie auch näher verwandt sind.

Die bebilderte Monographie der Gattung Dionysia (GREY-WILSON 1989) verschaffte dem Autor einen Eindruck der iranischen Landschaft, in der die in Gießen kultivierten Arten zu Hause sind.

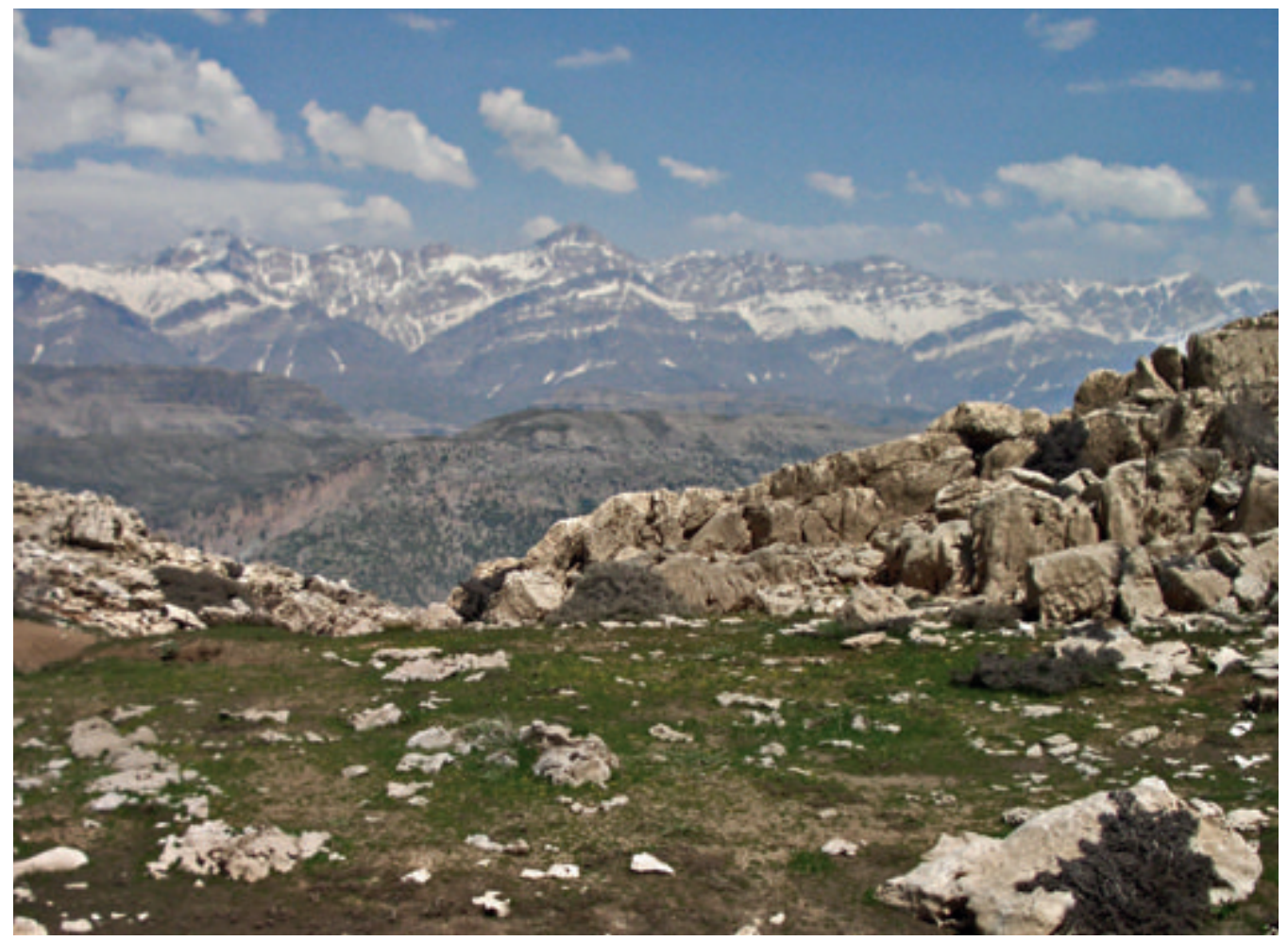

Abb. 1: Landschaft im Zagros-Gebirge. 

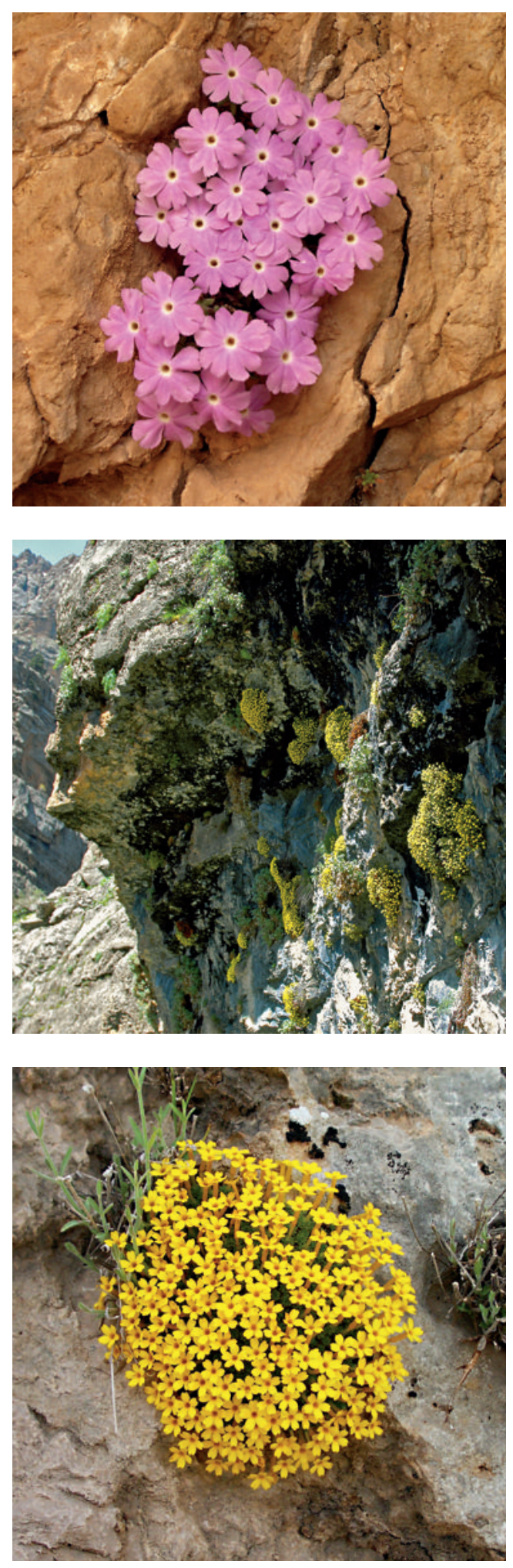

Aus floristischer Sicht wirkt das Gebiet zunächst nicht sehr interessant. Erst als der Autor Ende der 1990er Jahre von einer schwedischen Expedition und einer deutschen Reisegruppe hörte, die den Iran besuchten, wuchs das Interesse an diesem Gebiet. Wenn man die Pflanzen an ihrem Naturstandort gesehen hat, kann man im botanischen Garten die Kulturbedingungen den natürlichen Gegebenheiten viel besser angleichen.

Der Iran ist etwa fünf mal so groß wie Deutschland, die Hälfte davon ist Wüste oder wüstenartig. Im Iran gibt es rund 8000 Pflanzenarten. Davon sind 1700 endemisch, immerhin 22\% aller Pflanzenarten im Iran. Auf verschiedenen Reisen besuchte der Autor hauptsächlich den Westteil des Landes mit dem Zagros-Gebirge. Dies hat ungefähr die Ausmaße der Alpen, erstreckt sich aber von Nord nach Süd. Die höchsten Erhebungen sind der Zard Kuh (4558 m) und Kuh Dena (4404 m). Das Hochland, in dem man sich bewegen kann, liegt zwischen 1500 und 1800 m Höhe. Ungefähr $80 \%$ der Niederschläge fallen hier im Winter und Frühjahr. Das Gebirge besteht aus Kalkgestein und ist entsprechend durchlässig, aber auch speicherfähig, so dass die Flüsse und größeren Bäche ganzjährig Wasser führen und eine Bewässerung für die Landwirtschaft möglich ist. In südlicheren Flusstälern wird sogar Reis angebaut.

\section{Dionysia}

Zu Beginn der Reiseaktivitäten waren laut GREYWiLSON (1989) nur 27 Dionysia-Arten aus dem Iran bekannt, 13 davon nur von der Erstbeschreibung. Im Laufe der letzten Jahre hat sich ihre Zahl auf 35 erhöht (LidÉn 2008). Als ganz neue Taxa wurden D. zschummelii, D. zetterlundii, D. viva, D. crista-galli, D. tacamahaca und 2 neue Unterarten beschrieben. Dies zeigt, dass manche Gegenden botanisch noch nicht intensiv untersucht worden sind und sicher noch weitere Arten auf ihre Entdeckung warten. Dazu

Abb. 2 (oben): Dionysia archibaldii.

Abb. 3 (Mitte): Felshang mit Dionysia gaubae.

Abb. 4 (unten): Dionysia zagrica. 


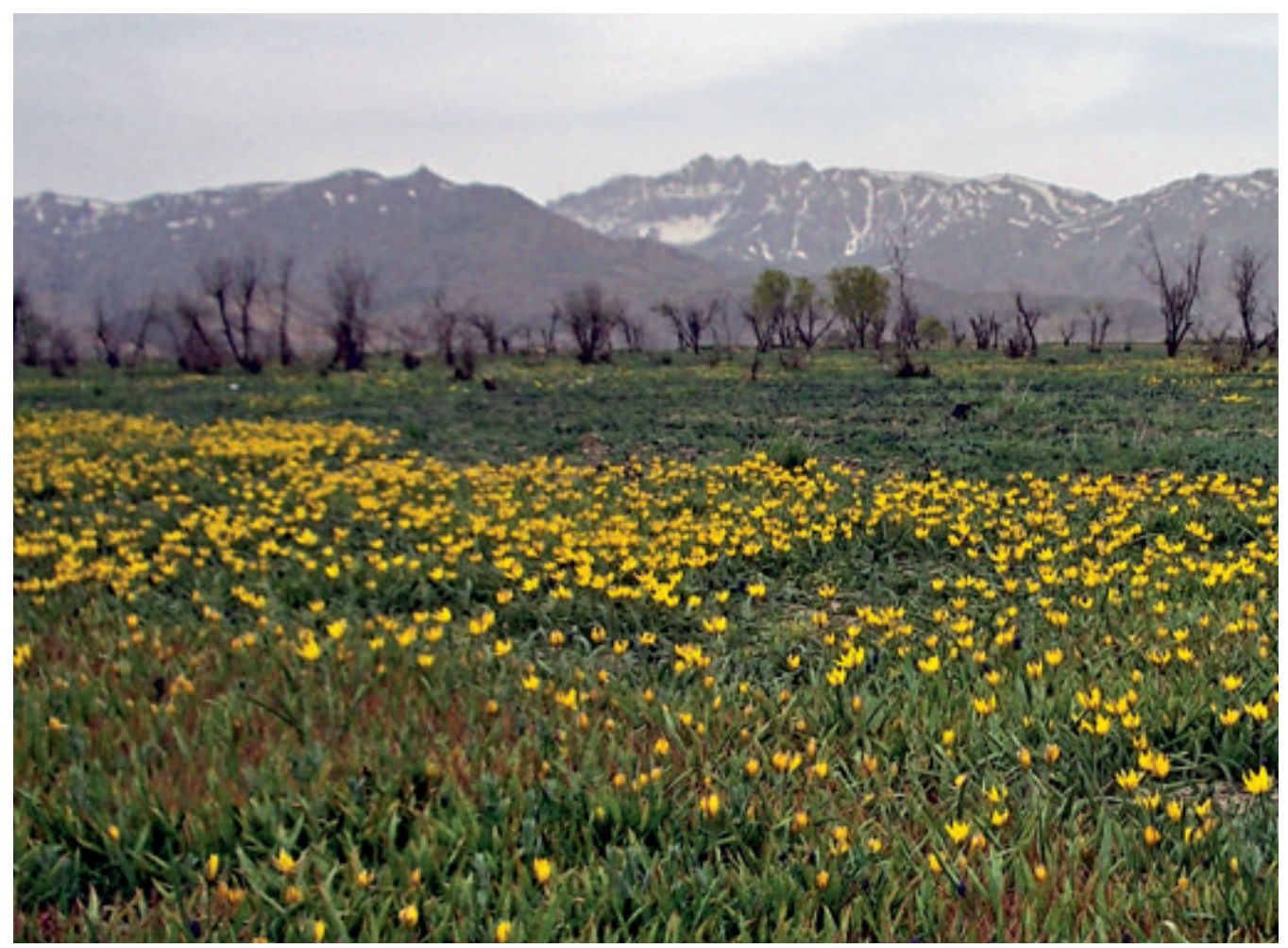

kommt, dass die Verbreitung vieler Arten nur auf einen einzigen Standort, einen Felsen oder einen Berg beschränkt ist. Auch all diese neu beschriebenen Dionysia-Arten sind bisher nur von einem Standort bekannt. Die meisten Wuchsorte befinden sich in nach Norden gerichteten und überhängenden Felswänden, in denen die Pflanzen vor Regen und direkter Sonneneinstrahlung geschützt sind. Solche speziellen Standorte sind meist nur von Dionysien belegt, wobei sogar bis zu drei Arten zusammen vorkommen können. Trotzdem sind bisher nur ganz wenige Naturhybriden bekannt. In Kultur hybridisieren alle Arten so stark miteinander, dass sie meist nicht rein erhalten werden können. In der Natur fällt auf, dass bei den dichtpolsterigen Arten nur etwa jede dritte Pflanze überhaupt einen guten Samenansatz hat, wofür es noch keine einleuchtende Erklärung gibt.

Abb. 5: Üppig blühender Bestand von Tulipa biebersteiniana.
Etliche Arten schützen sich vor zu starker Wasserabgabe durch dichte Behaarung, einen mehlartigen Belag, harzartige Ausscheidungen oder Einrollen der Blattränder. Ihre Blütenfarben sind verschiedene Gelb- oder Rosa-Lila-Töne. Auf botanischen Exkursionen ist es oft reizvoll, Standorte, an denen laut älterer Quellenangaben bestimmte Pflanzen gefunden wurden, aufzusuchen. Nicht immer ist dies erfolgreich. So wurden auch leider zwei Dionysia-Arten, die seit über 100 Jahren bekannt sind, nicht wiedergefunden.

\section{Geophyten}

Eine andere erwähnenswerte Pflanzengruppe sind die Geophyten oder Zwiebelpflanzen, vor allem die Lilien-, Erdrauch- und Aronstabgewächse. Einige dieser Pflanzen sind schon seit Jahrhunderten in unseren Gärten in Kultur wie beispielsweise Tulpe und Kaiserkrone. Tulpen haben eine recht große Verbreitung von Griechenland bis nach China. Die größte Artenzahl ist in Zentralasien anzutreffen. Im Iran kommen 24 Arten vor. Die häufigste Art ist die großblü- 

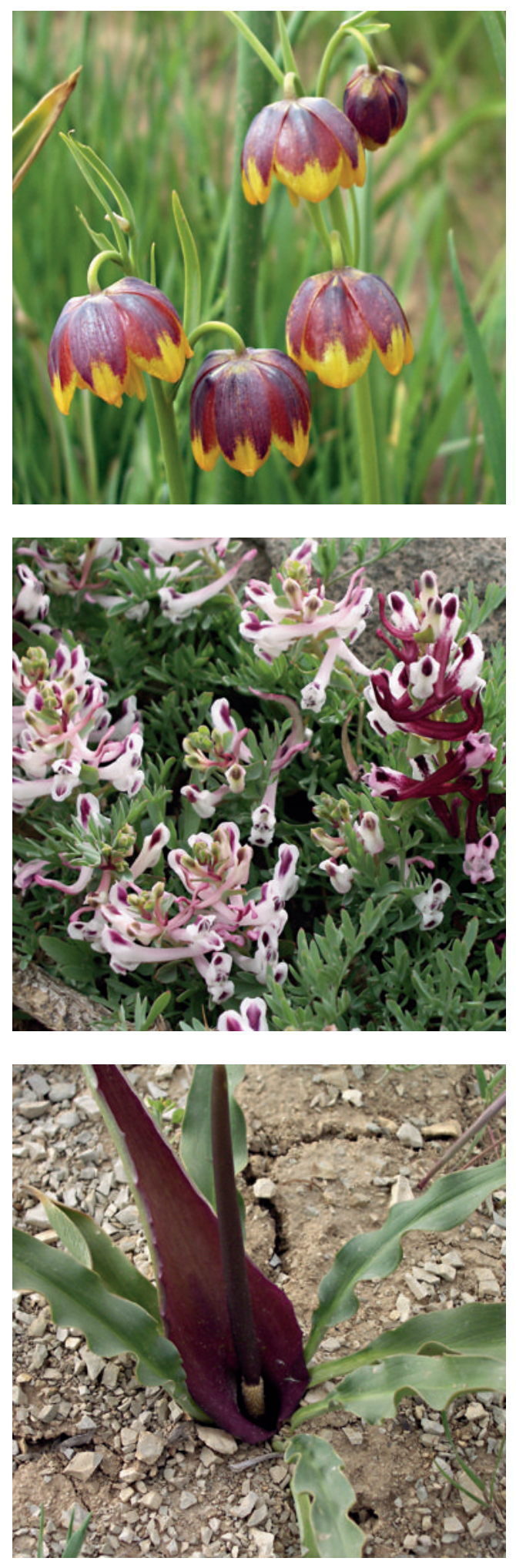

tige rote Tulipa systola. An feuchten Stellen und in Kulturflächen kommt die gelb blühende Tulipa biebersteiniana in Massenbeständen vor. Sie ähnelt unserer Weinbergs-Tulpe (T. sylvestris).

Schachbrettblumen (Fritillaria) sind in der gemäßigten nördlichen Zone einschließlich Amerika verbreitet. Im Iran gibt es 16 Arten. Die Kaiserkrone (Fritillaria imperialis) ist auf Iran, Afghanistan und Pakistan beschränkt. Dort wird sie schon lange in Gärten kultiviert, allerdings auch in der Türkei, von wo sie im 16. Jh. nach Holland kam. Der bekannteste Fundort im Iran ist eine Gegend namens Chelgerd im ZentralZagros. Während der Blütezeit von April bis Mai ist dieser Ort auch für Iraner ein beliebtes Ausflugsziel. Dann erscheinen ganze Hänge in Rot von Millionen Blüten. In der iranischen Sprache werden die Kaiserkronen wegen ihrer Form übersetzt „umgedrehte Tulpen“ genannt.

Nur eine weitere Fritillaria-Art erreicht die Größe der Kaiserkrone. Es handelt sich um Fritillaria persica mit grünlichgelben bis bräunlichen kleineren Blüten. In Kultur ist davon eine Variante aus der Türkei mit violettschwarzer Blütenfarbe. Eine kleine Art ist die auf alpine Schuttfluren im Zagros-Gebirge beschränkte F. zagrica. An extrem feuchten Stellen, manchmal sogar in Bächen, die aber im Sommer austrocknen, wächst $F$. reuteri.

An einem Standort in einer frühjahrsfeuchten Wiese wurde eine ganz braune, $40 \mathrm{~cm}$ hohe Schachbrettblume gefunden. In der Literatur ist eine solch gefärbte Art nur von der Grenze zu Aserbaidschan als F. grandiflora beschrieben, doch die Merkmale stimmten nicht überein. Die Beschreibung passte besser zu F. olivieri, aber nicht die Blütenfarbe, die allerdings auch kein relevantes Bestimmungsmerkmal ist. Vielleicht handelte es sich aber auch um eine noch nicht beschriebene Art.

Die bei uns aus Weinbergen bekannte blaue Traubenhyazinthe (Muscari) findet man im Iran in mehreren Arten recht häufig. Vor allem in

Abb. 6 (oben): Fritillaria reuteri.

Abb. 7 (Mitte): Corydalis verticillaris.

Abb. 8 (unten): Biarum straussii. 
feuchten Äckern wachsen sie in solchen Massen, dass man von Weitem meint, es wäre eine Wasserfläche.

Am Alvand Kuh bei Hamadan fallen besonders die Lerchensporne (Corydalis verticillaris) auf, die auch von diesem Berg zuerst beschrieben wurden. Am schönsten sind die purpur blühenden, außerdem gibt es noch weiße und alle Schattierungen. An jedem anderen Standort sind sie ein wenig verschieden, teils mit stärker gebogenem, teils mit geradem Sporn. Auch die Größe der Blüte variiert. Aufgrund solcher Unterschiede sind mehrere Unterarten beschrieben worden.

Auffällig sind die Aronstabgewächse vor allem, wenn sie blühen, auch wenn man bei näherer Betrachtung besser die Nase zuhält. Der Aasgeruch ist manchmal sehr penetrant. Er lockt Fliegen und Käfer für die Bestäubung an. Arum rupicola wird dort bis $1 \mathrm{~m}$ groß und hat meist eine dunkelpurpur oder seltener grünlich weiß gefärbte Spatha.

Im Norden nahe des Kaspischen Meeres gibt es eine Arum-Art, deren Blütenstand unserem heimischen Aronstab (A. maculatum) gleicht. Bisher wurde als östlichster Fundort die Krim in der Ukraine angegeben. Auch wird die Art in der Flora Iranica nicht erwähnt. $\mathrm{Ob}$ es sich tatsächlich um A. maculatum handelt und ob diese Art bereits für den Iran beschrieben wurde, müsste geprüft werden.

Im Frühjahr findet man gelegentlich Eminium, Aronstäbe mit dunkelpurpurnen Blütenständen. Sie fallen nicht so stark auf, da die Pflanzen zur Blütezeit auch Laubblätter haben und es noch andere Begleitpflanzen gibt. Ganz anders verhält es sich bei der verwandten Gattung Biarum, deren Blütenstände im Herbst erscheinen, wenn die Laubblätter noch nicht vorhanden sind. In dieser Zeit findet man kaum noch etwas Grünes, die Äcker sind längst abgeerntet, und dann stehen die dunkelrot leuchtenden Blütenstände wie rote Fähnchen in einer ausgedörrten Landschaft.

Abb. 9 (oben): Biarum carduchorum.

Abb. 10 (unten): Blütenstand von Eminium jaegeri.

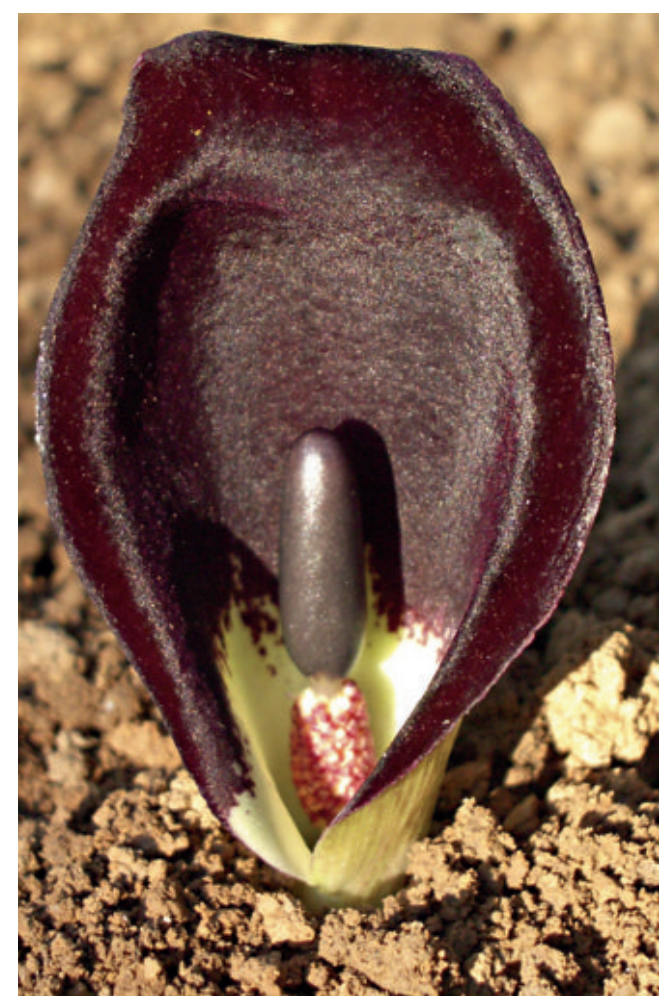




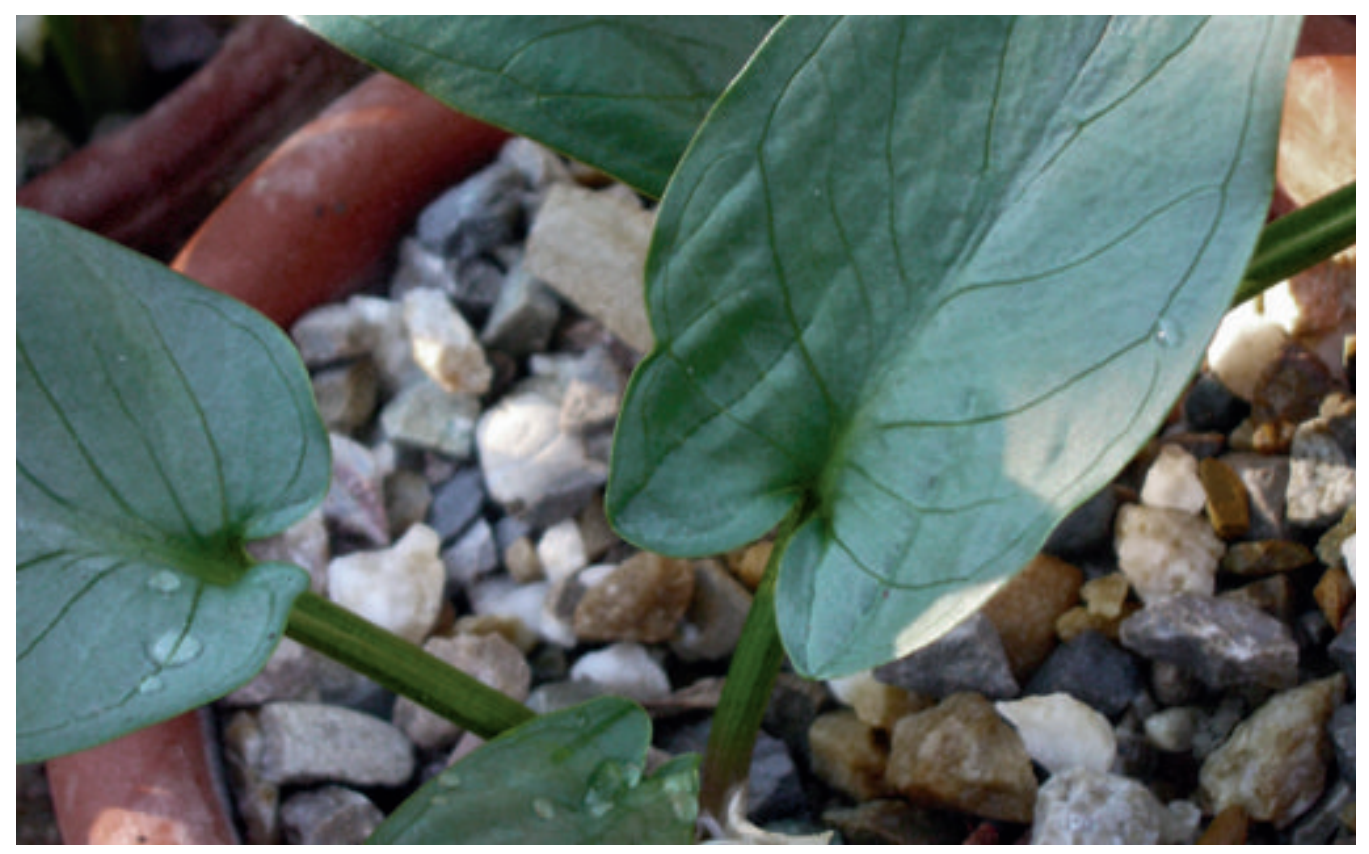

\section{Eine neue Eminium-Art}

Vor ein paar Jahren wurde im Frühjahr auf einer abgeweideten Fläche eine unbekannte Knollenpflanze mit einem für Araceen typischen Fruchtstand entdeckt. Aus Samen herangezogene Jungpflanzen wurden im botanischen Garten Gießen kultiviert. Ein paar Jahre lang brachte die Pflanze regelmäßig nur ganzrandige Blätter hervor, was zur der Vermutung verleitete, dass es sich um ein Biarum handeln könnte. Im Iran erscheinen im Herbst die Blütenstände von Biarum carduchorum. Da der Fruchtstand der unbekannten Aracee im Frühjahr erschienen war, musste sie ja im Herbst geblüht haben, was für Biarum spricht, wenn einem keine anderen herbstblühenden Araceen bekannt sind.

Endlich zeigte sich tatsächlich im Herbst ein Blütenstand. Es war jedoch kein Biarum carduchorum. Das Nachbestimmen anhand verschiedener Floren (Flora Iranica, Flora of Iraq, Flora of Turkey), führte nicht zum Erfolg. Bei Biarum sowie Eminium stimmten immer ein paar Merkmale und ein paar nicht, als ob es eine Mischung von beiden wäre.

Deshalb wurde Josef Bogner, AraceenSpezialist vom Botanischen Garten München, zu Rate gezogen. Auch er wunderte sich über die
Merkmale, die eigentlich zu 2 Gattungen passten. Die Spatha ist vorne verwachsen, ein gattungstypisches Merkmal für Biarum, wofür eigentlich auch die Blütezeit spricht. Allerdings ist der Kolben kurz und dick. Eine molekulargenetische Untersuchung bestätigte dann, dass es sich um ein Eminium handeln muss. Mit ihrer Blütezeit im Herbst ist die Art eine Ausnahme innerhalb der sonst im Frühjahr blühenden Eminium-Arten. Das sollte nicht irritieren, denn innerhalb der Gattung Biarum gibt es auch als Ausnahme ein im Frühjahr blühendes Biarum straussii.

Josef BOGNer beschrieb die Art als Eminium jaegeri Bogner \& Boyce (Bogner \& Boyce 2008). Typische Merkmale für diese Art sind z. B. die flach-kugelige Knolle und die an der Spitze zurückgeschlagene Spatha. Der Kolben ist deutlich kürzer als die Spatha. Die Beeren enthalten einen eiförmigen, bis $6 \mathrm{~mm}$ langen Samen. Bis jetzt ist diese neue Art nur von einem einzigen Standort bekannt. Dieser befindet sich in einer Höhe von 1800 m auf der Westseite des Kuh Rang Bergzuges im Zagros-Gebirge im Nord-

Abb. 11: Blätter von Eminium jaegeri. 


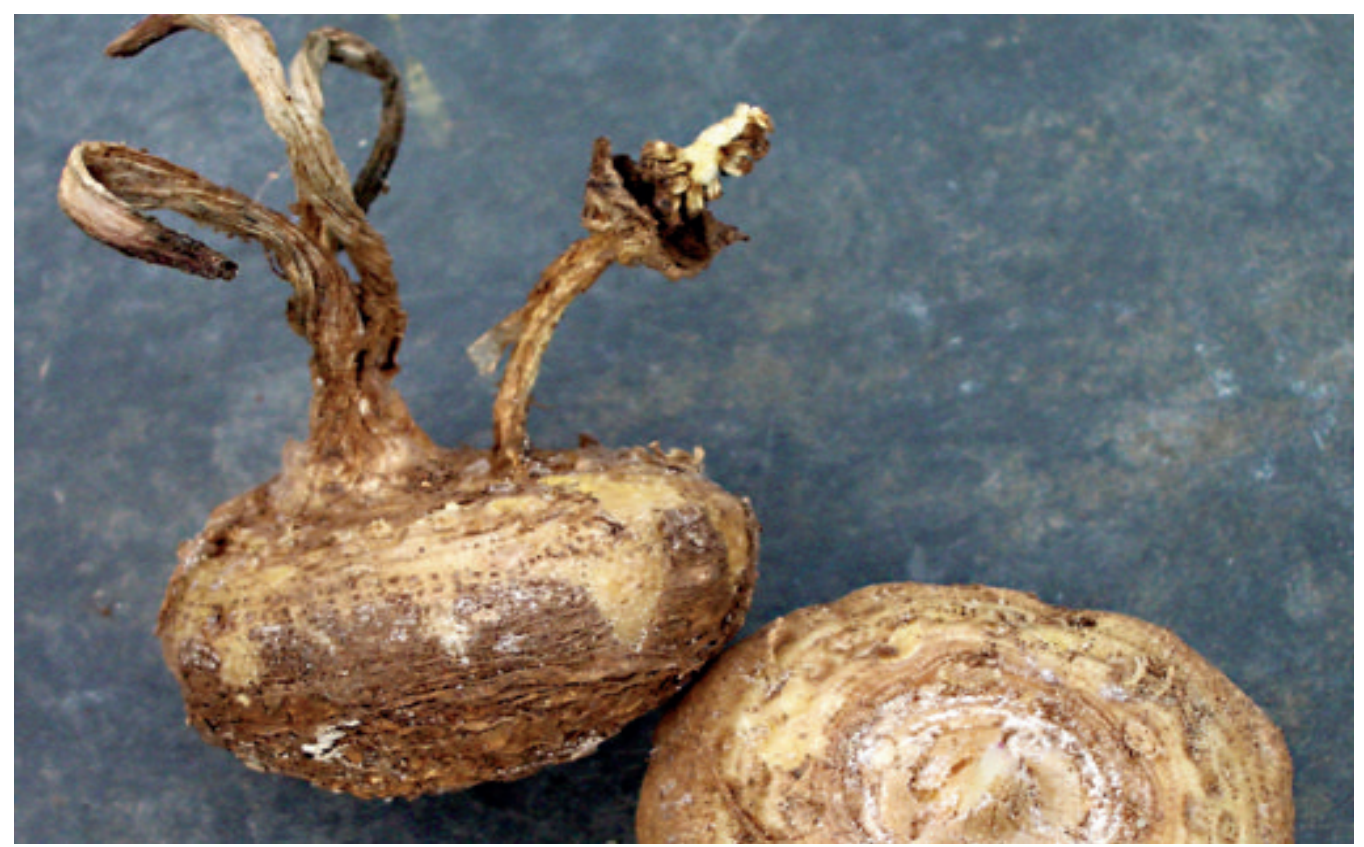

west-Iran, Provinz Bakhtiari. Er ist ein süd- bis südwestlich geneigter Hang mit vereinzelten Eichenbäumen. Begleitpflanzen gibt es wegen der starken Beweidung kaum. Der Boden ist steindurchsetzter Lehm über Kalkstein-Untergrund.

Frischer Pollen wurde an die Universität Wien geschickt, um Oberflächenstrukturen zu untersuchen. Dort wurde vor kurzem entdeckt, dass einige Aronstabgewächse als Baustoff der Pollenwand nicht das übliche Sporopollenin bilden, sondern Polysaccharide.

\section{Viel Neues zu entdecken}

In der iranischen Flora werden vermutlich auch neue Allium-Arten entdeckt. REINHARD FrITSCH (IPK Gatersleben) ist noch mit der Aufarbeitung der iranischen Lauch-Arten beschäftigt. Erwähnenswert ist eine neue Art aus der Sektion Melanocromyum. Ihre Blütenblätter sind grün bis rosa, der Fruchtknoten ist dunkelrot gefärbt.

Ein weiterer Neufund gelang mit einer Orchidee. In über 2500 m Höhe wurde eine RagwurzOrchidee (Ophrys) entdeckt. Dies war der höchste Standort überhaupt, an dem die Ex- kursionsgruppe Orchideen fand. Sie sind im Iran sonst eher in feuchteren Tallagen anzutreffen. Der Orchideen-Spezialist J. GoELz bestimmte sie umgehend als Ophrys transhyrcana. Es müssen ihm aber Zweifel aufgekommen sein, denn als er im darauffolgenden Jahr in den Iran reiste, besuchte er auch diesen Standort. Dabei stellte sich heraus, dass diese Ragwurz doch eine neue Art ist. Die Beschreibung erfolgte als Ophrys zagrica GÖLZ, benannt nach dem Fundort und der Verbreitung im Zagros-Gebirge.

\section{Literatur}

Bogner, J. \& Boyce, P. 2008: Eminium jaegeri (Araceae), a new species from northwestern Iran. -

Willdenowia 38: 149-153.

GreY-Wilson, C. 1989: The genus Dionysia. - Woking.

LIDÉn, M. 2008: The genus Dionysia (Primulaceae),

a synopsis and five new species. -

Willdenowia 37: 37-61.

\section{Internetseite}

http://geb.uni-giessen.de/geb7volltexte/2009/7152/pdf/

Sdf_2009_01_26_31.pdf

Spiegel der Forschung 2009, Uni Gießen. 